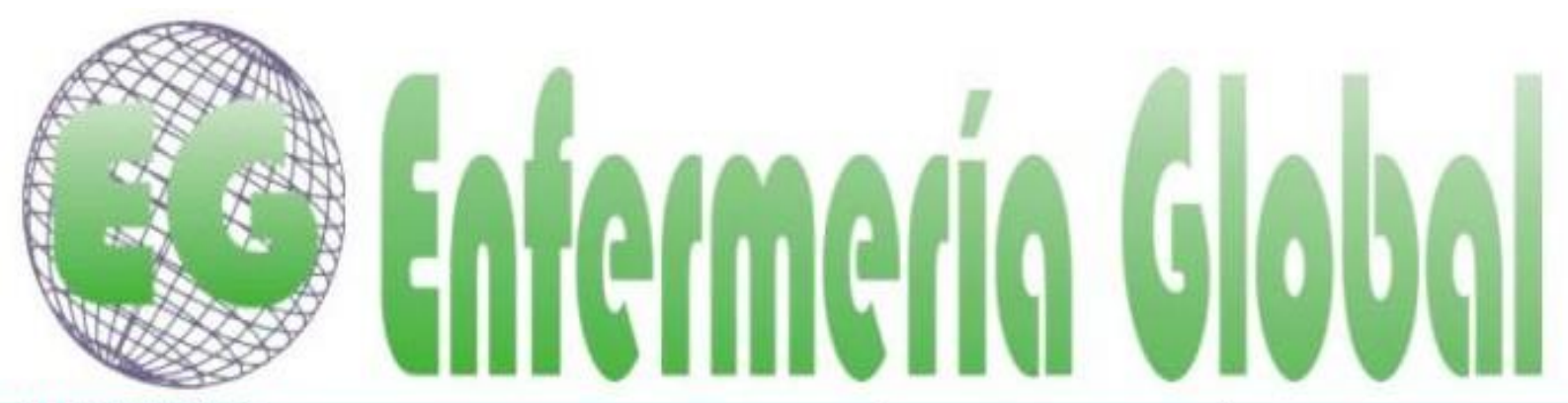

\title{
Incidencia de Flebitis asociada a Catéteres Centrales de Inserción Periférica en UCI Adultos: Implementación de un Protocolo para Enfermería
}

Phlebitis Incidence Associated to Peripherally Inserted Central Catheters in Adults ICU: Implementation of a Nursing Protocol

*Yaniz Álvarez, Francisco Javier *Ajona Martínez-Polo, Sara *Díaz Arozarena, Encarna *Senar Senar, Juan Bautista *Garralda Etxarri, Nacho *Morales Villanueva, Asier ** de la Rosa Fernández-Pacheco, Pedro Antonio *DUE en UCl. E-mail: $\quad$ fjya@hotmail.es $\quad * * M I R$ Medicina Preventiva y Salud Pública. Complejo Hospitalario de Navarra. España.

\section{http://dx.doi.org/10.6018/eglobal.16.1.248081}

\section{RESUMEN}

Justificación: La flebitis es una de las complicaciones mas frecuentes de los Catéteres Centrales de Inserción Periférica. La evidencia científica sobre la utilidad de las escalas de medición para el diagnostico de flebitis es escasa.

Objetivos: Comparar la incidencia de flebitis antes y después de la implementación de un protocolo.

Material y métodos: Estudio de cohortes retrospectivo en 159 pacientes ingresados en UCI, a los que se les ha colocado un PICC, en dos periodos equivalentes de dos años consecutivos, Periodo 1 $(n=59)$; frente a un grupo del Periodo $2(n=100)$ en el que se aplicó un nuevo protocolo para el diagnóstico y manejo de flebitis (definición de flebitis, aplicación de la Visual Infusion Phlebitis Score y valoración continua).

Resultados: El riesgo de ser diagnosticado de flebitis fue significativamente menor en el P2 (OR: 0.09 , I.C.95\% 0.01-0.52)

Conclusiones: La incorporación del protocolo redujo un 90\% los diagnósticos de flebitis.

Palabras clave: Catéter Central de Inserción Periférica; Flebitis: Visual Infusion Phlebitis Score; Protocolo; UCI 


\section{ABSTRACT}

Phlebitis incidence related to peripherally inserted central catheters (PICCs): New nursing protocol application. Phlebitis is one of the most common complications of peripherally inserted central catheters (PICCs). The scientific evidence about the utility of measuring scales to phlebitis diagnosis is very limited.

Objectives: To compare phlebitis incidence rate before and after the introduction of a new protocol.

Materials and Methods: Retrospective cohort study in 159 patients admitted to the intensive care unit (ICU) for two years. First we assess phlebitis in 59 patients (group 1). After that we apply a new protocol to identify phlebitis with visual score and continuous evaluation to 100 patients (group 2)

Results: The probability of being diagnosed of phlebitis is significantly smaller in intervention group (OR: 0.09; 95\% C.I.: 0.02-0.57).

Conclusions: Protocol application reduced the diagnosis of phlebitis in $90 \%$

Keywords: Peripherally inserted central catheters (PICCs,) Phlebitis, Visual Infusion Phlebitis Score, Protocol, ICU.

\section{INTRODUCCIÓN}

Los catéteres venosos centrales (CVCs) constituyen una parte integral del paciente crítico ${ }^{1}$. Tanto los CVCs como los catéteres centrales de inserción periférica (PICCs), son necesarios para la administración de agentes terapéuticos múltiples que requieren acceso venoso fiable por un periodo largo de tiempo. Algunos de ellos, como la quimioterapia, nutrición parenteral o soluciones hipertónicas pueden ser administrados de manera segura solo a través del sistema venoso central ${ }^{2}$. También son necesarios para la monitorizaciónn hemodinámica y la extracción frecuente de muestras de sangre ${ }^{3,4}$, situaciones que se dan continuamente en el ámbito de los cuidados intensivos.

La popularidad de los catéteres PICCs es cada vez mayor debido a que presentan varias ventajas con respecto a los CVCs. En concreto, los PICCs son más fáciles de insertar ${ }^{5}$, producen menos dolor en la inserción ${ }^{6}$ y su extracción conlleva menos riesgos $^{7}$, siendo una alternativa segura y eficaz ${ }^{8}$. Además, tienen menor incidencia de sepsis relacionada con el catéter, y son más cómodos para el paciente respecto a otros catéteres centrales ${ }^{6,9}$. De este modo, se minimizan otras complicaciones graves asociadas a la inserción del CVC como el hemotórax y el neumotórax ${ }^{10,11}$.

Sin embargo, a pesar de sus ventajas, los PICCs pueden presentar complicaciones como la mala posición del catéter, trombosis, flebitis, hemorragia, daño en tendones o nervios, bacteriemia, arritmias cardiacas y dolor en el pecho ${ }^{6,7}$. De todas estas complicaciones, la flebitis es una de las más frecuentes ${ }^{7}$. En este sentido, parece ser que el riesgo de flebitis, así como el riesgo de infección, se minimiza utilizando PICCs de poliuretano frente a catéteres de silicona ${ }^{12}$. Del mismo modo, la técnica de inserción del catéter de Seldinger modificada minimiza el trauma del tejido y aumenta la probabilidad de lograr un acceso venoso con una sola aguja y menos complicaciones ${ }^{13}$. Además, es conocido que el calibre del catéter y el lugar de inserción ${ }^{2}$ son factores que se asocian a un mayor riesgo de flebitis en estos catéteres, no viéndose influenciados por el tipo de soluciones que se infunde a través de ellos. 
Por último, en las unidades de cuidados intensivos (UCls), la utilización de catéteres multilumen es relativamente frecuente y su mayor grosor conlleva un mayor riesgo de flebitis $^{2}$. Existen estudios que valoran la efectividad de protocolos de inserción de PICCs liderados por especialistas en enfermería clínica en la reducción de complicaciones como flebitis, tromboflebitis, etc ${ }^{14}$. Sin embargo, existe poca evidencia científica en relación al diagnóstico de flebitis asociada a PICCs en pacientes hospitalizados en unidades de cuidados intensivos. Una revisión sistemática realizada en 2014 demostró que existen muchas escalas de flebitis pero ninguna ha sido validada para su uso en la práctica clínica ${ }^{15}$. Esto es importante porque según qué escala de flebitis se utilice la incidencia de la misma varía considerablemente ${ }^{2}$. La Visual Infusion Phlebitis (VIP) Score $^{16}$ es una escala habitualmente utilizada para el diagnóstico y tratamiento de la flebitis en catéteres venosos periféricos, pero no se dispone evidencia sobre su aplicación en pacientes con PICCs.

La flebitis puede ser de origen mecánico o infeccioso. La incidencia de flebitis de origen infeccioso ha disminuido drásticamente desde la implantación del protocolo de Bacteriemia Zero ${ }^{17}$. La flebitis de origen mecánico puede comenzar con cualquier trauma en la vena provocando cambios endoteliales que activan los sistemas de coagulación, fibrinolítico y del complemento, entre otros. La activación de estos sistemas produce una disminución del flujo sanguíneo y una respuesta inflamatoria que a su vez causa hipersensibilidad y enrojecimiento del sitio de inserción, pudiendo llegar a complicaciones trombóticas o incluso infecciosas. Por tanto, la consecuencia es un aumento de los días de estancia hospitalaria y un aumento del coste sanitario, sin olvidar la incomodidad que supone para el paciente la retirada y reinserción de un nuevo catéter ${ }^{2}$. Por ello, el desarrollo de valoraciones e intervenciones que reduzcan este problema constituye una necesidad para los profesionales de enfermería.

El presente estudio tiene los siguientes objetivos:

a. Comparar la incidencia de flebitis antes y después de la implementación de un nuevo protocolo estandarizado que incluye la escala VIP como herramienta de diagnóstico de flebitis en dos periodos distintos: Periodo 1 (P1): Octubre de 2012-Febrero de 2013 y Periodo 2 (P2): Octubre de 2013Febrero de 2014.

b. Conocer la incidencia de flebitis causadas por PICCs y analizar los factores relacionados con su desarrollo en pacientes hospitalizados en la UCI del Complejo Hospitalario de Navarra.

c. Comparar la incidencia de flebitis entre los catéteres de 2 luces versus los catéteres de 3 luces.

\section{MATERIAL Y MÉTODOS}

El estudio se desarrolló en una UCI polivalente de 12 camas del Complejo Hospitalario de Navarra, cuyas especialidades de referencia son cirugía plástica, cirugía maxilofacial y ginecología, siendo la mayoría de los ingresos de patología médica (55\%). La unidad presenta una distribución longitudinal con 10 boxes abiertos y 2 cerrados para aislamientos.

Se definió un protocolo de observación, diagnóstico y manejo de flebitis, impartiendo formación sobre el mismo al personal de enfermería de la unidad. Este protocolo consistía en: 
1. Diagnóstico de flebitis según los criterios de la VIP Score $(1998)^{16}$.

2. Valoración y seguimiento del punto de inserción del PICC en cada turno.

3. En aquellos casos con una puntuación de 2 puntos en la escala VIP SCORE, se aplicaron medidas higiénicas y de vigilancia para ver si la inflamación remitía o evolucionaba a flebitis (3 o más puntos en la escala), en cuyo caso se retiraba el catéter (ver Tabla l)

Tabla I: VIP Score para la detección y tratamiento de la flebitis.

\begin{tabular}{|c|c|c|c|}
\hline Número & Signos & Intervenciones & $\begin{array}{l}\text { Modificaciones para el } \\
\text { estudio }\end{array}$ \\
\hline $\mathbf{0}$ & $\begin{array}{l}\text { No se observa ningún } \\
\text { signo de dolor o infección. }\end{array}$ & $\begin{array}{l}\text { No hay signos de } \\
\text { flebitis. Observar el } \\
\text { punto de inserción. }\end{array}$ & \\
\hline 1 & $\begin{array}{l}\text { Dolor/eritema en torno al } \\
\text { lugar de inserción. }\end{array}$ & $\begin{array}{l}\text { Aparición de los } \\
\text { primeros signos de } \\
\text { flebitis. Observar el } \\
\text { punto de inserción. }\end{array}$ & \\
\hline 2 & $\begin{array}{l}\text { Se observan dos de los } \\
\text { siguientes signos: } \\
\text { Dolor a lo largo del } \\
\text { catéter. } \\
\text { Eritema } \\
\text { Inflamación }\end{array}$ & $\begin{array}{l}\text { Signos de flebitis. } \\
\text { Remplazar el catéter. }\end{array}$ & $\begin{array}{l}\text { Posibles signos de } \\
\text { flebitis. } \\
\text { Calor local. } \\
\text { Elevación de la } \\
\text { extremidad. } \\
\text { Valoración continuada. }\end{array}$ \\
\hline 3 & $\begin{array}{l}\text { Se observa: } \\
\text { Dolor a lo largo del } \\
\text { catéter. } \\
\text { Eritema. } \\
\text { Inflamación. }\end{array}$ & $\begin{array}{l}\text { Flebitis instaurada. } \\
\text { Remplazar el catéter y } \\
\text { considerar tratamiento. }\end{array}$ & \\
\hline 4 & $\begin{array}{l}\text { Se observa: } \\
\text { Dolor a lo largo del } \\
\text { catéter. } \\
\text { Eritema. } \\
\text { Endurecimiento. } \\
\text { Vena palpable. }\end{array}$ & $\begin{array}{l}\text { Flebitis avanzada y/o } \\
\text { inicio de } \\
\text { tromboflebitis. } \\
\text { Remplazar el catéter y } \\
\text { considerar tratamiento. }\end{array}$ & \\
\hline 5 & $\begin{array}{l}\text { Se observa: } \\
\text { Dolor a lo largo del } \\
\text { catéter. } \\
\text { Eritema. } \\
\text { Endurecimiento. } \\
\text { Vena palpable. } \\
\text { Fiebre. }\end{array}$ & $\begin{array}{l}\text { Estado avanzado de } \\
\text { tromboflebitis. } \\
\text { Iniciar tratamiento. } \\
\text { Remplazar el catéter. }\end{array}$ & \\
\hline
\end{tabular}

Traducido y adaptado de Jackson A. (1998)

Para valorar la incidencia de flebitis antes y después de incorporar el protocolo en la unidad, se llevó a cabo un estudio de cohortes retrospectivo entre dos grupos de pacientes ingresados en periodos equivalentes de dos años consecutivos: Periodo 1 
(P1), de octubre a febrero de 2012/2013; Periodo 2 (P2), de octubre a febrero de 2013/2014.

Los datos se recogieron a través de los documentos del registro de enfermería y de las historias clínicas informatizada del $\mathrm{CHNa}$, seleccionando los pacientes a los que se les había realizado la inserción de uno o más PICCs durante este periodo.

En ambos periodos, todos los PICCs fueron insertados por el personal de enfermería, con técnica Seldinger, utilizando catéteres de poliuretano y siguiendo las precauciones estándar recomendadas del Proyecto Bacteriemia Zero ${ }^{17}$. La elección de la vena se hizo por familiaridad y confianza de la enfermera para realizar la colocación del PICC. La ubicación del catéter siempre fue confirmada por la radiografía de tórax. El tipo de catéter y el número de luces se decidió en función de su uso terapéutico. Para el cuidado y mantenimiento de los PICCs se siguieron los protocolos de enfermería del hospital basados en el proyecto Bacteriemia Zero ${ }^{17}$.

Las observaciones para el diagnóstico de flebitis fueron realizadas por tres enfermeros, unificando criterios diagnósticos según el protocolo para disminuir la variabilidad en los resultados. Se realizó una revisión diaria de la gráfica de enfermería donde se registran las variables del estudio y la observación del punto de punción de los PICCs insertados.

Las variables estudiadas fueron: edad, sexo, criterio de gravedad del paciente según APACHE II ${ }^{18}$, vena canalizada (basílica o cefálica), PICC de dos o tres luces, fecha de inserción, días de colocación, fecha de retirada del catéter o fecha de alta de la unidad y presencia flebitis asociada a PICC (sí o no). Se consideraron casos de flebitis aquellos que presentaban 3 o más puntos en la escala VIP Score. Además, en aquellos casos con una puntuación de 2 en la escala (dos de los tres signos: dolor, eritema e inflamación), las intervenciones de enfermería iban dirigidas al seguimiento y la valoración por turno para prevenir o vigilar su progresión.

Para realizar la evaluación y planificación de esta actividad en el servicio, puesto que está dentro del plan de seguridad del hospital, se pidió permiso a los responsables de la unidad, recibiendo su aprobación por escrito. Los datos se trataron con total confidencialidad.

El estudio recibió aprobación por parte del servicio de investigación del CHNa.

Para el análisis de los datos se utilizó el paquete estadístico STATA 12.

Las variables categóricas se expresan mediante la distribución de frecuencias, tanto globales como específicas, del grupo de pacientes con inserción de PICC. Las variables continuas se describen mediante la media y la desviación estándar. Se estableció un nivel de significación estadística a dos colas de $\alpha=0,05$.

Se calculó las diferencias de la distribución de variables entre los periodos de estudio mediante un análisis univariante utilizando la prueba de Chi-cuadrado para variables categóricas y t de Student para variables cuantitativas.

Asimismo, para valorar la interacción entre la incidencia de flebitis y las variables de interés según el periodo de estudio, se realizó un análisis multivariable de regresión logística estratificando por periodo de estudio y ajustando por sexo, edad, y aquellas 
variables que mostraron una asociación estadísticamente significativa en el modelo univariante.

Por último, para hallar la asociación entre la flebitis y los catéteres de 2 y 3 luces en ambos periodos se utilizó el test de Chi-cuadrado.

\section{RESULTADOS}

En el P1 y en P2 se recogieron los datos de 59 y 100 pacientes respectivamente.

La distribución de las variables cualitativas y cuantitativas se muestra en la Tabla II.

Tabla II: Distribución de variables en la muestra según el periodo de estudio.

\begin{tabular}{|c|c|c|c|c|c|}
\hline Variable & Categoría & Periodo 1 & Periodo 2 & Total & Valor $\mathbf{p}$ \\
\hline \multirow{2}{*}{$\operatorname{Sexo}(\%)$} & Hombre & $38(64,41)$ & $64(64)$ & $102(64,15)$ & 0,959 \\
\hline & Mujer & $21(35,59)$ & $36(36)$ & $57(35,85)$ & \\
\hline \multirow{4}{*}{ Cuartiles de edad (\%) } & $17-52$ & $9(15,25)$ & $32(32)$ & $41(25,79)$ & 0,055 \\
\hline & $53-63$ & $21(35,59)$ & $20(20)$ & $41(25,79)$ & \\
\hline & $66-76$ & $15(25,42)$ & $26(26)$ & $41(25,79)$ & \\
\hline & $77-91$ & $14(23,73)$ & $22(22)$ & $36(22,64)$ & \\
\hline \multirow{2}{*}{ Tipo catéter (\%) } & 2 luces & $45(76,27)$ & $54(54)$ & $99(62,26)$ & 0,005 \\
\hline & 3 luces & $14(23,73)$ & $46(46)$ & $60(37,74)$ & \\
\hline \multirow{4}{*}{ APACHE II (\%) } & $1-11$ & $17(28,81)$ & $35(35)$ & $52(32,7)$ & 0,366 \\
\hline & $12-16$ & $10(16,95)$ & $22(22)$ & $32(20,13)$ & \\
\hline & $17-23$ & $13(22,03)$ & $23(23)$ & $36(22,64)$ & \\
\hline & 24-38 & $19(32,2)$ & $20(20)$ & $39(24,53)$ & \\
\hline \multirow{2}{*}{ Flebitis (\%) } & No & $50(84,75)$ & $98(98)$ & $148(93,08)$ & $<0,001$ \\
\hline & Sí & $9(15,25)$ & $2(2)$ & $11(6,91)$ & \\
\hline $\operatorname{Edad}\left(\mathrm{DE}^{*}\right)$ & & $\begin{array}{c}64,6 \\
(13.99)\end{array}$ & $\begin{array}{c}61,57 \\
(17,72)\end{array}$ & $\begin{array}{c}62,51 \\
(16,47)\end{array}$ & 0,217 \\
\hline APACHE II (DE*) & & $\begin{array}{l}18,27 \\
(9,13)\end{array}$ & $\begin{array}{l}16.71 \\
(8.13)\end{array}$ & $\begin{array}{c}17,29 \\
(17,29)\end{array}$ & 0,265 \\
\hline $\begin{array}{l}\text { Duración de catéter } \\
\text { (DE*) }\end{array}$ & & $6.59(6,0)$ & $7.41(6,55)$ & $7,11(6,38)$ & 0,474 \\
\hline
\end{tabular}

*DE: Desviación estándar

Se encontraron diferencias estadísticamente significativas en la distribución de ambos periodos de los tipos de catéteres en la incidencia de flebitis, estando la edad en el límite de significación estadística. Los participantes en el P1 $(n=59)$ presentaron las siguientes características demográficas: 38 (64,41\%) fueron hombres y 21 (35,58\%) 
mujeres y la edad estaba comprendida entre 32 y 87 años con una media de 64,60 años; la gravedad de los pacientes de acuerdo al APACHE II se situaba en un rango de 3 a 45; de los catéteres insertados, el 76,27\% (45) fueron de 2 luces y el 23,73\% (14) fueron de 3 luces; la duración media de los catéteres fue de 6,59 días y en este periodo se diagnosticaron 9 casos de flebitis (15,25\%).

En el P2 $(n=100)$ las características demográficas de los participantes fueron las siguientes: el $64 \%$ fueron hombres y el $36 \%$ mujeres; la edad estaba comprendida entre 17 y 90 años con una media de 61,57 años; la gravedad de los pacientes de acuerdo al APACHE II se situaba entre puntuaciones de 1 a 38; de los 100 PICCs insertados, el $54 \%$ fueron de 2 luces y el $46 \%$ de 3; la duración media de los catéteres insertados fue de 7,41 días y durante el P2 se realizaron 2 diagnósticos de flebitis (2\%). Estos datos, tanto del P1 como del P2, se muestran en la Tabla II.

La Tabla III recoge las Odds Ratios para flebitis de las variables de estudio estratificadas por periodos.

Tabla III. Asociación de flebitis con las variables de estudio en ambos periodos.

\begin{tabular}{|c|c|c|c|c|c|c|}
\hline \multirow[b]{3}{*}{ Variable } & \multicolumn{6}{|c|}{ Flebitis } \\
\hline & \multicolumn{2}{|c|}{ Valor $\mathbf{P} \mathbf{C h i}^{2}$} & \multicolumn{2}{|c|}{ Odds Ratio } & \multicolumn{2}{|c|}{$\begin{array}{c}\text { valor } \mathbf{p} \text { (Intervalo de } \\
\text { Confianza) }\end{array}$} \\
\hline & $\begin{array}{c}\text { Periodo } \\
1\end{array}$ & $\begin{array}{c}\text { Periodo } \\
2\end{array}$ & $\begin{array}{c}\text { Periodo } \\
1\end{array}$ & $\begin{array}{c}\text { Periodo } \\
2\end{array}$ & Periodo 1 & Periodo 2 \\
\hline Sexo1 & 0,363 & 0,284 & 1,70 & ---- & $\begin{array}{c}0,371(0,26- \\
11,25)\end{array}$ & ---- \\
\hline Edad $^{2}$ & 0,377 & 0,551 & 0,95 & 0,96 & $\begin{array}{c}0,359(0,88- \\
1,02)\end{array}$ & $\begin{array}{c}0,398(0,84- \\
1,04)\end{array}$ \\
\hline $\begin{array}{c}\text { Tipo } \\
\text { catéter }^{3}\end{array}$ & 0,113 & 0,909 & 2,46 & 0,96 & $\begin{array}{c}0,125(0,38- \\
16,01)\end{array}$ & $\begin{array}{c}0,909(0,05- \\
19,93)\end{array}$ \\
\hline $\begin{array}{l}\text { APACHE } \\
\text { II }^{4}\end{array}$ & 0,006 & 0,651 & 2,90 & 0,99 & $\begin{array}{l}0,022(1,8- \\
7,13)\end{array}$ & $\begin{array}{c}0,024(0,30- \\
3,08)\end{array}$ \\
\hline
\end{tabular}

1: Sexo masculino frente al femenino. 2: Cuantitativa. 3: Cateteres de 3 vías frente al de 2 vías. 4: Escala Apache en cuarteles. Variable cuantiativa. Todas las variables están ajustadas por las otras variables de la tabla.

No se hallaron diferencias estadísticamente significativas en las Odds Ratios de las variables de estudio según periodo. La magnitud de las Odds Ratio de las variables APACHE y tipo de catéter varió considerablemente de un periodo a otro, no siendo estas diferencias significativamente estadísticas.

Durante el P2 se observó una baja incidencia de flebitis en nuestra unidad $(2 \%$ frente a $15.25 \%$ del P1), no hallando diferencias estadísticamente significativas de mayor incidencia de flebitis en catéteres de dos luces frente a los de tres $(p=0,602)$. De igual manera, en el P1 tampoco se encontraron diferencias estadísticamente significativas 
de mayor incidencia de flebitis en catéteres de dos luces frente a los de tres $(p=0,424)$.

La Tabla IV muestra las Odds Ratio del las variables del estudio ajustando por periodo.

Tabla IV. Asociación de flebitis con las variables de estudio ajustando por periodos.

\begin{tabular}{|c|c|c|c|c|}
\hline Variable & $\mathbf{O R}$ & Valor p & \multicolumn{2}{|c|}{ Intervalo de confianza al $95 \%$} \\
\hline $\operatorname{Sexo}^{1}$ & 2,66 & 0,262 & 0,48 & 14,60 \\
\hline Edad $^{2}$ & 0,96 & 0,081 & 0,91 & 1,01 \\
\hline $\begin{array}{l}\text { Tipo de } \\
\text { catéter }^{3}\end{array}$ & 1,73 & 0,479 & 0,38 & 7,86 \\
\hline APACHE II ${ }^{4}$ & 1,99 & 0,035 & 1,05 & 3,80 \\
\hline $\begin{array}{l}\text { Periodo de } \\
\text { estudio }^{5}\end{array}$ & 0,087 & 0,007 & 0,01 & 0,52 \\
\hline
\end{tabular}

Se encontraron diferencias significativas en el diagnóstico de flebitis al comparar los periodos 1 y 2 ( $p=0,001)$, es decir, que el riesgo de ser diagnosticado de flebitis fue significativamente menor en el P2 (OR: 0,09; I.C. 95\%: 0,02-0,57) frente al P1.

Las diferencias de las Odds Ratio de tener flebitis según la puntuación de la escala APACHE II o el tipo de catéter empleado variaron en magnitud al estratificar según periodo o al ajustar por periodo de estudio, lo cual sugiere una posible interacción entre el periodo de estudio y el diagnóstico de flebitis según estas variables.

No se encontraron diferencias significativas al analizar otros factores relacionados con la flebitis como la edad, el sexo y el APACHE II. Finalmente, al analizar las 159 inserciones de PICCs en ambos periodos, se observó que existía una asociación estadísticamente significativa entre la gravedad del paciente (APACHE II con puntuación más alta) y la colocación de catéteres de 3 luces $(\mathrm{p}=0,013)$.

\section{DISCUSIÓN:}

Este es el primer estudio que valora la utilización de la escala VIP Score para el manejo de PICCs. La utilización de PICCs en la práctica clínica está volviéndose muy común en las UCls, debido a su fácil inserción y a su baja incidencia de complicaciones en comparación con $\mathrm{CVC}^{7,10,11}$. 
Se ha descrito en la literatura que el PICC es un método eficaz y seguro en pacientes ingresados en la $\mathrm{UCl}$ y constituye una excelente alternativa para la administración de medicamentos y soluciones por períodos prolongados ${ }^{19}$.

El presente estudio aporta algo de evidencia en la experiencia en el uso de PICCs en pacientes adultos y el diagnostico preciso de flebitis mediante el uso de la escala diagnóstica VIP Score, sin encontrar ningún hallazgo concluyente en relación a una mayor incidencia de flebitis en los catéteres de tres luces con respecto a los de dos luces. Un estudio de casos y controles realizado por Mazzola et al. ${ }^{2}$ encontró una asociación entre el calibre del catéter y el riesgo de flebitis. En nuestro estudio, los catéteres de tres luces y dos luces tienen un diámetro de 2,4 mm... y 2,3 mm respectivamente, por lo que la ausencia de diferencias estadísticamente significativas podría estar debida a que la diferencia del calibre entre ambos tipos de catéteres es de tan sólo 0,1 mm.

Por un lado, se insertaron más catéteres de tres luces cuanto mayor era la gravedad del paciente según el APACHE II. Esto es comprensible ya que cuanto mayor sea la gravedad del paciente necesitará más tratamientos y una monitorización más invasiva ${ }^{3}$, lo cual favorece la decisión de utilizar catéteres con más luces. Además, el estado más grave de estos pacientes requieren la utilización de medicamentos múltiples más agresivos que por sí mismos podrían ser factor de riesgo de flebitis ${ }^{2}$. Por otro lado, la reducción de la Odds Ratio de flebitis según catéter utilizado o puntuación en escala APACHE II del periodo 2 pudo ser causada porque las medidas de higiene y observación pautadas por el protocolo de la unidad pudo ser realizadas con especial cuidado en los pacientes más graves o con catéteres de 3 luces, lo cual explicaría la posible interacción entre esas variables y el periodo de estudio para el riesgo de flebitis.

En cuanto al diagnóstico de flebitis, los datos muestran que tras la implantación del protocolo en el P2 hay una marcada disminución de los casos diagnosticados de flebitis. Esta reducción en la incidencia de flebitis puede deberse a varios factores: En primer lugar, la formación específica que recibió el staff de enfermería sobre el uso de la VIP Score pudo ayudar a concienciar a los profesionales sobre los riesgos de flebitis, lo que pudo causar mejoras no observadas en las técnicas de inserción, cuidados y tipo de catéter del segundo periodo con respecto al primero. Por otro lado, al tratarse de un estudio retrospectivo y recoger los datos de la historia clínica informatizada, los datos pueden estar mal clasificados, sobre todo por una posible pérdida de información relacionada con las variables de estudio en P1 debido a un registro incompleto de su seguimiento en la grafica de enfermería al no existir protocolo de observación. Este hecho sesgaría nuestros resultados hacia el valor nulo por no estar recogidas todas las posibles flebitis de ese periodo; sin embargo, a pesar de esta limitación, se han encontrado diferencias estadísticamente significativas entre ambos periodos. En segundo lugar, no podemos excluir que la disminución en la incidencia de flebitis haya sido debida a factores desconocidos en ambos periodos de estudio, pese a haber escogido dos periodos de estudio equivalentes en el calendario. En tercer lugar, la modificación que realizamos en la escala VIP Score, indicando no retirar los catéteres hasta que se obtuviese una puntuación de 3 en la escala y vigilar y cuidar las vías con una puntuación de 2, pudo haber causado la disminución de la incidencia de diagnósticos de flebitis por un cambio en la clasificación de las mismas, puesto que es posible que en el periodo 1 se diagnosticaran flebitis que hubieran obtenido 2 puntos en la escala de VIP Score. Se ha descrito en la literatura que en ocasiones pueden encontrarse signos inflamatorios y eritema en el punto de inserción 
que pueden confundirse con flebitis, sobre todo cuando se trata de venas más superficiales y de menor calibre. Con un una valoración continuada por parte del personal de enfermería y tratamiento con calor y elevación de la extremidad, estos signos pueden desaparecer sin mayores complicaciones ${ }^{2}$.

La formación del personal de enfermería en el uso de la escala VIP Score y su posterior utilización evita potenciales sobrediagnósticos de flebitis que conllevan la retirada de PICCs y posterior canalización de otros catéteres sustitutivos, disminuyendo la iatrogenia asociada a la canalización de PICCs, el gasto sanitario subsiguiente, y las cargas de trabajo, lo cual deriva en un aumento de la calidad y seguridad de los cuidados ${ }^{20}$.

Como recomendación para futuras investigaciones, sería conveniente realizar una validación en el uso de esta escala en el manejo de PICCs.

\section{CONCLUSIONES}

A lo largo del estudio se ha objetivado una disminución de la incidencia de flebitis entre el P1 y el P2 y ninguna diferencia significativa con los factores relacionados estudiados.

Así mismo, no se encuentran ninguna asociación entre la incidencia de flebitis y la inserción de catéteres de tres luces frente a los de dos.

La aplicación de un protocolo con intervenciones como la definición de flebitis, la VIP score y la valoración continuada por parte del personal de enfermería, reducen considerablemente los casos diagnosticados de flebitis, posiblemente por disminución del sobrediagnóstico. Se necesitan más estudios para profundizar en estos hallazgos. El proyecto de reciente creación de Flebitis Zero supone una oportunidad de estudio de la flebitis en este tipo de catéteres ${ }^{21}$.

\section{REFERENCIAS}

1. Fitchie C. Central venous catheter-related infection and dressing type. Intensive Crit Care Nurs. 1992;8(4):199-202.

2. Mazzola JR, Schott-Baer D, Addy L. Clinical factors associated with the development of phlebitis after insertion of a peripherally inserted central catheter. Journal of Intravenous Nursing.1999;22(1):36 - 42.

3. Gomez Palomar C, Miquel Perez T. Catéter venoso central de inserción periférica con múltiples luces: una buena opción para monitorizaciónn de presión y tratamiento. Enferm Cardiol. 2009; XVI(47-48):99-103.

4. Chlebicki MP, Teo EK . Review of peripherally inserted central catheters in the Singapore acute-care hospital. Singapore Med J. 2003; 44(10):531-35.

5. Cotogni P, Pittiruti M. Focus on peripherally inserted central catheters in critically ill patients. World J Crit Care Med. 2014;3(4):80-94.

6. Miyagaki H, Nakajima K, Hara J, Yamasaki M, Kurokawa Y, Miyata H, Takiguchi S, Fujiwara Y, Mori M, Doki Y. Performance comparison of peripherally inserted central venous catheters in gastrointestinal surgery: a randomized controlled trial. Clin Nutr. 2012; 31(1):48-52.

7. Harter C, Ostendorf T, Bach A, Egerer G, Godschmidt H, Ho AD. Peripherally inserted central venous catheters for autologous blood progenitor cell transplantation in patients with haematological malignancies. Support Care Cancer. 2003;11(12):790-4 
8. Vivien R Griffiths, Peter Philpot. Peripherally inserted central catheters (PICCs): do they have a role in the care of the critically ill patients. Intensive and critical care nursing 2002; 18(1): 37-47.

9. Di Giacomo, M. Comparison of three peripherally-inserted central catheters: pilot study. British Journal of Nursing. 2009; 18(1):8-16.

10. Hata Y, Morita S, Morita Y, Awatani T, Takasaki M, Horimi T, Ozawa Z. Peripheral insertion of a central venous access device under fluoroscopic guidance using a peripherally accessed system (PAS) port in the forearm. Cardiovasc Intervent Radiol.1998; 21(3):230-3.

11. Stephen C, Loughran and Marcello Borzatta, M .Peripherally Inserted Central Catheters: A Report of 2506 Catheter Days. J Parenter Enteral Nutr.1995; 19(2):1336.

12. Ong CK, Venkatesh SK, Lau GB, Wang SC. Prospective randomized comparative evaluation of proximal valve polyurethane and distal valve silicone peripherally inserted central catheters. J Vasc Interv Radiol. 2010; 21(8): 1191 - 6.

13. Nichols I, Humphrey JP. The efficacy of upper arm placement of peripherally inserted central catheters using bedside ultrasound and microintroducer technique. J Infus Nurs. 2008; 31(3):165-76.

14. Park JY, Kin HL. A comprehensive review of clinical nurse spcialist-led peripherally inserted central catheter placement in Korea: 4101 cases in a tertiary hospital. J. Infus Nursing 2015; 38(2):122-8

15. Gillian Ray-Barruel, Denise F. Folit. Infusion phlebitis assessment measures: a systematic review. Journal of evaluation in clinical practice 2014; 20(2): 191-202.

16. Seguridad del Paciente. Proyecto Bacteriemia Zero. [Acceso 20 Diciembre de 2014]. Disponible en:http://www.seguridaddelpaciente.es/es/proyectos/financiacionestudios/proyecto-bacteriemia-zero/

17. Knaus WA.APACHE II. Final form and national validation results of a severity of disease classification system. Crit Care Med.1984; 12(3):213-63.

18. Jackson A. Infection control: a battle in vein infusion phlebitis. Nursing Times. 1998; 94(4):68-71.

19. Ng PK, Ault MJ, Maldonado LS. Peripherally inserted central catheters in the intensive care unit. J Intensive Care Med.1996; 11(1):49-54.

20. Ferrete-Morales C, Vázquez-Pérez M A, Sánchez-Berna M; Gilabert-Cerro I, CorzoDelgado $J \mathrm{E}$, Pineda-Vergara $\mathrm{J} A$, et al. Incidence of phlebitis due to peripherally inserted venous catheters: impact of a catheter management protocol. Enferm Clin. 2010;20(1):3-9.

21. Proyecto flebitis Zero. [Acceso 18 de junio de 2015]. Disponible en:http://flebitiszero.com/app/

Recibido: 15 de enero de 2016;

Aceptado: 01 de abril de 2016

ISSN 1695-6141

() COPYRIGHT Servicio de Publicaciones - Universidad de Murcia 